\title{
Metodologías colaborativas y participativas de aprendizaje para el alumnado. El cuento intergeneracional como recurso educativo
}

\section{Collaborative and participatory learning methodologies for students. The intergenerational story as an educational resource}

\author{
jasa2@um.es, silviana@um.es,pma8@um.es \\ Departamento Teoría e Historia de la Educación \\ Universidad de Murcia \\ Murcia, España
}

Juan Antonio Salmerón Aroca, Silvia Martínez De Miguel López, Pedro Moreno Abellán

\begin{abstract}
Resumen- Este trabajo muestra un proyecto de innovación educativa implementado en el ámbito de la educación superior. Tiene como propósito la elaboración de una serie de relatos (cuentos) destinados fundamentalmente, al alumnado del Grado en Educación Social, pero con intencionalidad de extender su influencia a las personas mayores, en una ineludible conexión intergeneracional. Para ello, se utilizaron estrategias cualitativas para el aprendizaje colaborativo, que fomentan un aprendizaje significativo, experiencial y cooperativo, vinculados con las competencias de la titulación. Se pretende de esta manera, ampliar las capacidades y realizaciones profesionales del alumnado, integrando valores culturales y sociales sobre la madurez, que favorezcan la convivencia intergeneracional y la inclusión social. Los resultados de la experiencia desarrollada se muestran a través de vivencias y elaboraciones de relatos de diversa índole, creados de manera individual, pero involucrando a las personas mayores en un proceso creativo intergeneracional con temáticas que pertenecen a su experiencia, y que ofrecen una imagen de la vejez, no discriminatoria hacía el colectivo de personas mayores. Para finalizar, conjuntamente a la lectura de cada relato, se abordaron en el aula mediante asamblea, los aspectos metodológicos y curriculares del trabajo realizado, que sirvieron como elementos de mejora del Grado en Educación Social.
\end{abstract}

Palabras clave: innovación educativa, educación superior, métodos de enseñanza, educación social, personas mayores

Abstract- This work shows a project of educational innovation implemented in the field of higher education. Its purpose is the development of a series of stories (tales) intended primarily for the students of the Degree in Social Education, but with the intention of extending its influence to the elderly, in an unavoidable intergenerational connection. For this, qualitative strategies for collaborative learning were used, which promote a significant, experiential and cooperative learning, linked to the skills of the degree. In this way, it is intended to expand the capabilities and professional achievements of students, integrating cultural and social values about maturity, which favor intergenerational coexistence and social inclusion. The results of the experience developed are shown through experiences and elaborations of different kinds of stories, created individually, but involving the elderly in an intergenerational creative process with themes that belong to their experience, and that offer an image of old age, non-discriminatory towards the group of elderly people. Finally, together with the reading of each story, the methodological and curricular aspects of the work carried out were addressed in the classroom through assembly, which served as elements for improving the Degree in Social Education.

Keywords: educational innovation, high education, teaching methods, social education, old people

\section{INTRODUCCIÓN}

La configuración del Espacio Europeo de Educación Superior exige, a partir de la Declaración de Bolonia, profundizar a través de la investigación en metodologías alternativas a las tradicionales (Delgado y De Justo, 2018). Bajo este marco, el propio título de la propuesta que se presenta, lo coloca en la esfera del ámbito de las prácticas de innovación social, que consideran que estas acciones poseen un efecto favorable para las personas, agregando valor social (Jarré, 2016). Entendiendo de esta manera, que la innovación social puede configurarse tanto desde los fines que persigue, como desde los medios que utiliza, con la finalidad de hacer frente a problemas o retos sociales (Ramos, 2017).

Es por ello que, animados tras la obtención del premio internacional Europeo, SiforAGE, en el año 2014 a la mejor innovación social por el proyecto intergeneracional desarrollado en la Universidad de Murcia (Martínez De Miguel, Salmerón y Moreno, 2018), se determinó poder dar continuidad, y extrapolarlo a otros contextos educativos. Así, el desafío que se propone para la actividad realizada, se articula en torno a la necesidad de una educación intergeneracional (Bedmar y Montero, 2003), desde la óptica de que, educar para reducir problemas de comunicación y estereotipos intergeneracionales, es educar en favor de una sociedad para todas las edades y la solidaridad intergeneracional (Sánchez, et al., 2007). Se lleva a cabo, con la finalidad de profundizar sobre las relaciones intergeneracionales, llevando a cabo el proyecto de una manera participativa, tras realizar una actualización de los núcleos de interés, y observar una óptica variada de intervenciones, ya sea tanto a nivel académico, como a nivel institucional (Salmerón, Martínez De Miguel y Belchí, 2018).

En este sentido, este proyecto surge con la intención de contribuir no sólo a la creación de un material docente (cuentos educativos), realizado de forma colaborativa con la 
máxima implicación del alumnado a través del uso de técnicas cualitativas, sino también la promoción del pensamiento, la reflexión crítica, la interrelación del conocimiento, la comprensión de realidades diferentes (personas mayores), y el desarrollo de capacidades y competencias múltiples, vinculadas a las competencias profesionales. De esta forma, el cuento se considera una buena herramienta pedagógica. Según Tamayo Valdés y León Pérez (2017) al estudiar las aportaciones didácticas de los cuentos, señalan que, despierta la afición a la lectura; aporta estímulos lúdicos; desarrolla el lenguaje semántico, fonológico y narrativo en el alumnado. Así, se asume el valor del cuento como elemento fundamental para aprender sin las connotaciones negativas que tradicionalmente se han tenido sobre las personas mayores.

De la misma manera, se entiende que el uso de relatos hábilmente organizados, refuerzan la información, y dan al texto escrito, además del aspecto de un cuento, el de un manual, dirigido a aquellos profesionales que se dedican a la atención a personas mayores, o la implicación de acciones intergeneracionales con colectivos de mayores a través de los centros sociales destinados a estos colectivos, y en los programas de las Aulas Senior.

Como se ha expuesto, el proyecto ofrece como elaboración por parte del alumnado, una historia narrada en la que los personajes principales ofrezcan una visión de su vida, que ayude a romper falsas creencias y estereotipos sobre la vejez, con un enfoque ya no terapéutico sino educativo, a través de estrategias de trabajo colaborativo. Este enfoque innovador, posibilita la involucración de la propia persona mayor, así como también la de los profesionales que desarrollan los programas en los centros sociales participantes.

De esta manera, la atención a los aspectos motivacionales configura un aspecto esencial para el trasfondo cultural y científico con el que se han elaborados los textos. A la luz de estas ideas, y bajo el perfil puramente de los estereotipos negativos contra la vejez, existe la necesidad contrastada, de abordarlos en los centros sociales de mayores, tanto de talleres, como de proyectos intergeneracionales (Martínez De Miguel, Salmerón, y Escarbajal De Haro, 2017; Salmerón, Escarbajal De Haro y Martínez De Miguel, 2018).

\section{CONTEXTO}

La necesidad de la realización de esta experiencia fue puesta de manifiesto desde la coordinación del título de Educación Social, quién por medio de una evaluación cualitativa, el alumnado valoró ciertos aspectos relacionados con la docencia (guía docente, sesiones teóricas, sesiones prácticas, participación del alumnado en clase, tutorías presenciales y virtuales, examen, logro de competencias por parte del alumnado, trabajos propuestos en el marco de la asignatura, exigencia y cumplimiento de las recomendaciones transversales que se llevan a cabo desde la coordinación del título, etc.). En este sentido, el alumnado, valoró positivamente la introducción de una metodología menos memorística y más motivadora, acercamiento a la práctica profesional, y como aspectos generales de mejora, la densidad de contenidos, y un mayor trabajo interasignaturas. Desde esta perspectiva, se estableció una alianza de colaboración entre las asignaturas de Desarrollo Comunitario y Animación sociocultural, con la asignatura de Educación de Personas mayores, que se hizo extensivo a asignaturas del Máster de exclusión e inclusión social, para el desarrollo conjunto de actividades, que pudieran tener conexión y continuidad a lo largo del año académico.

Así, el programa de intervención se desarrolló en virtud de la resolución realizada por el rector de la Universidad de Murcia, a través de la convocatoria para promover proyectos y acciones de innovación y mejora en la Universidad de Murcia para el curso 2018/2019, y fue auspiciado por el profesorado del Grado de Educación Social de la Facultad de Educación de la Universidad de Murcia. En este sentido se consideró que las estrategias cualitativas para el aprendizaje colaborativo (Martínez de Miguel y Escarbajal de Haro, 2009), en combinación con técnicas de escritura narrativa, eran eficaces para fomentar un aprendizaje significativo, experiencial y cooperativo, toda vez que tienen su incorporación en las distintas competencias de la titulación, para indagar en las necesidades educativas, y desarrollar acciones con personas o grupos que puedan sufrir exclusión social o en riesgo de vulnerabilidad. Específicamente, se ha desarrollado en el Tercer curso del Grado de Educación Social, con el propósito de profundizar con el alumnado en metodologías colaborativas $\mathrm{y}$ participativas en relación con personas mayores, como futuros usuarios de sus servicios. La decisión de elaborar el proyecto con este alumnado, surge ante las oportunidades de innovación docente consensuadas con los propios alumnos, y contaron con la aprobación tanto del Consejo del departamento de Teoría e Historia de la Educación, como de la Dirección del Grado, profesores, tutores y coordinadores de $3^{\circ}$ curso.

El primer objetivo de este trabajo se relacionó con las Guías Docentes de las asignaturas, donde se persigue aplicar una metodología participativa y significativa para el alumno en torno a una realidad social profesionalizante. De esta manera, se obtuvo el permiso del centro social de personas mayores Murcia II, a través de sus coordinadores y directores, contando con la aprobación de los usuarios del centro, para poder intervenir con ellos, y que tomaron parte activa durante todas las fases del proyecto. El segundo objetivo, la creación de materiales docentes colaborativos que favorezcan la motivación por el aprendizaje, contó con la instrucción previa sobre la realización de relatos para el alumnado que decidió participar en la puesta en funcionamiento del mismo. El tercer objetivo planteado, consistió en la evaluación de la utilización de estrategias cualitativas intergeneracionales en enseñanza superior por parte del alumnado, con una clara intención profesionalizadora. Finalmente, el cuarto objetivo, integrar valores culturales sobre la madurez, que favorezcan la convivencia intergeneracional, fue elaborado a partir de la medición de estereotipos negativos hacia la vejez, antes y después de la intervención, con grupo experimental y grupo de control, cuyos resultados no se incluyen en este trabajo puesto que la baremación continua abierta a lo largo del primer semestre del año, concluyendo con la finalización del curso académico.

\section{DESCRIPCIÓN}

El proyecto estableció una experiencia intergeneracional entre personas mayores y estudiantes del Grado de Educación social. Favorece un intercambio de aprendizajes, y se logra como resultados del aprendizaje, una innovación en intervención social, basada en la colaboración activa y la participación comunitaria con un evidente trasfondo 
educativo. La práctica sirve para promover el conocimiento mutuo de ambas generaciones, generar compromiso y valores, como el respeto y la prosocialidad.

En la práctica, se ha planteado al alumnado la posibilidad de adaptar y utilizar la técnica de elaboración de relatos de vida, como metodología de investigación cualitativa, a partir de las vivencias de las personas mayores, tal y como describe Martín García (1995). De tal forma, el alumnado convirtió la biografía de cada persona mayor, obtenida mediante el uso de entrevistas en profundidad (semiestructurada con preguntas abiertas), en relatos de vida. Se basan en los acontecimientos personales de sus amplias trayectorias vitales (experiencias personales, familiares, laborales, etc.). Para ello, se siguió el procedimiento establecidos por Bolívar, Domingo y Fernández (2001), y se actuó de la siguiente manera; en primer lugar, y una vez realizado el diseño del trabajo de investigación, se procedió a la recogida de información mediante el trabajo de campo, y toma de contacto entre el alumnado y las personas mayores. En segundo lugar, se llevaron a cabo las entrevistas mediante una acción retrospectiva desde el momento actual de la persona mayor hasta su infancia, a través de un discurso interactivo. Se organizaron en función de las diferentes etapas del ciclo vital por los que han ido transitando sus vidas. En tercer lugar, a partir de la información obtenida, y registrada en soportes electrónicos sobre el curso de sus vidas, se procedió a su posterior transcripción, codificación, y análisis de contenido. Finalmente, se organizaron las principales ideas obtenidas, dando sentido al relato de vida construido.

Una vez obtenido el relato de vida se entiende que, el alumnado, ya posee una información sustancial necesaria para el proceso cognitivo y emocional de narración creativa. A partir de este punto, se procedió a la elaboración del cuento intergeneracional. Para ello, se les proveyó de técnicas para la construcción de microrrelatos y cuentos. De manera concreta, la técnica de escritura narrativa que se empleó para el desarrollo del escrito, fue la de la escritura creativa y gramática de la fantasía, dirigida a personas mayores en riesgo de exclusión social (ACCEM, 2018). Así, el alumnado construyó una historia breve y original, vertebrada por la estructura clásica para la elaboración de cuentos: planteamiento, nudo y desenlace, que se desarrolló a través de las propuestas del trabajo de Rodari (2016).

De esta manera, en primer lugar cada alumno construyó un personaje principal de la acción, en base a la investigación de campo realizada; definieron su biografía; crearon su perfil fisonómico (sexo, edad, constitución física, apariencia, etc.); sociológico (ocupación, educación, vida familiar, entretenimientos, etc.); y psicológico (frustraciones, temperamento, habilidades, etc.). Posteriormente se concretaron el resto de personajes, que intervendrían en la historia. En segundo lugar, se creó la escena (contexto, circunstancias dadas, deseos, conflictos personales u objetales, etc.). A continuación, se describió el conflicto principal (consciente o insconsciente), que mueve la escena, y finalmente, su desenlace.

De tal forma, cada alumno generó un relato, que fue matizándose, corrigiéndose y mejorándose, en función de las propias aportaciones de las personas mayores. De hecho, se convirtieron en los verdaderos protagonistas de las historias, y se involucraron de tal modo en la acción, que el relato definitivo fue elaborado de una manera concelebrada o en tándem, entre estudiantes y personas mayores. Todo ello, con motivo de los encuentros, que se fueron produciendo por el establecimiento de alianzas entre alumnos y personas mayores.

El desarrollo global del trabajo desarrollado por parte del alumnado se organizó a través de tutorías docentes en el aula con el profesorado, en combinación con la inmersión en el trabajo de campo. Para ello el alumnado tuvo que desplazarse hasta el centro de personas mayores Murcia II. De esta manera se llevaron a cabo seis sesiones de tutorización docente: dos iniciales para evaluación, explicación y entrega de materiales, así como para la realización del análisis de las opciones educativas que brindaba el proyecto, vinculándolo al currículum del educador social; dos sesiones intermedias de carácter formativo para la realización del seguimiento del trabajo del alumnado; y dos finales, a modo de evaluación sumativa, donde se llevó a cabo la asamblea final. Se analizaron los aspectos de formatos y forma de los elementos utilizados, y se revisaron los diarios de campo cumplimentados. Además, la asamblea permitió poner de relieve las inquietudes surgidas entre todos los participantes, y sirvió de evaluación para la trazabilidad del proyecto de forma global.

De la misma manera, se desarrollaron seis sesiones de trabajo de campo. Fueron distribuidas con la siguiente estructura: $1^{\mathrm{a}}$ Análisis de la realidad en el centro de personas mayores Murcia II; con la finalidad de conocer al grupo de con los que se iba a desarrollar el proyecto, así como la realización y cohesión de equipos de trabajo.
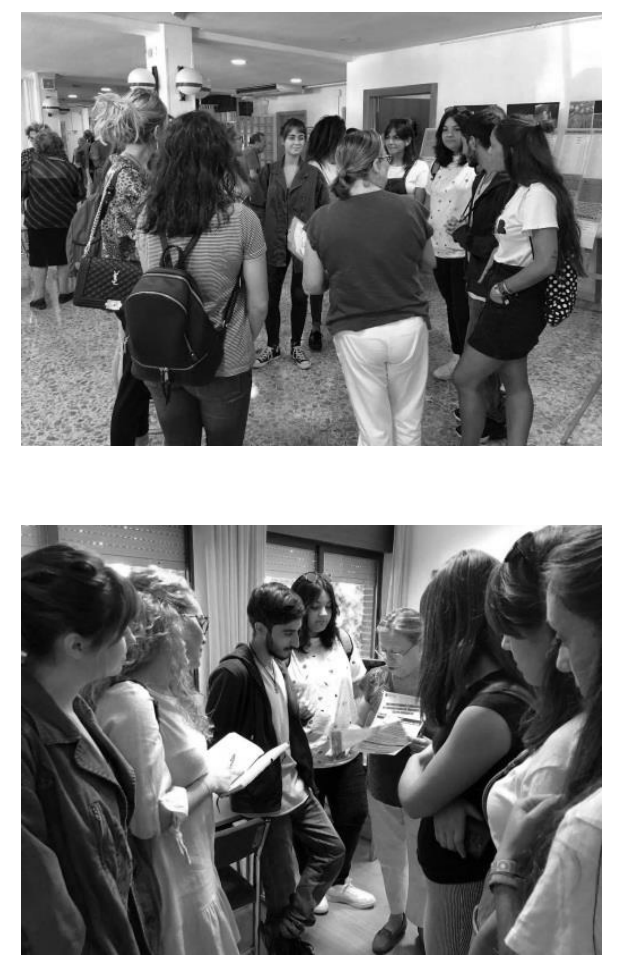

Figuras 1y 2. Construcción y cohesión del grupo de trabajo inicial.

$2^{a}$ Profundización en el análisis de la realidad del personaje elegido; para lo cual se llevó a cabo técnicas de dinamización con la persona mayor para la favorecer la reflexión, así como las entrevistas a través del dialogo persona a persona. De esta manera se favorecía la construcción del personaje, a través de la elaboración del relato de vida. 


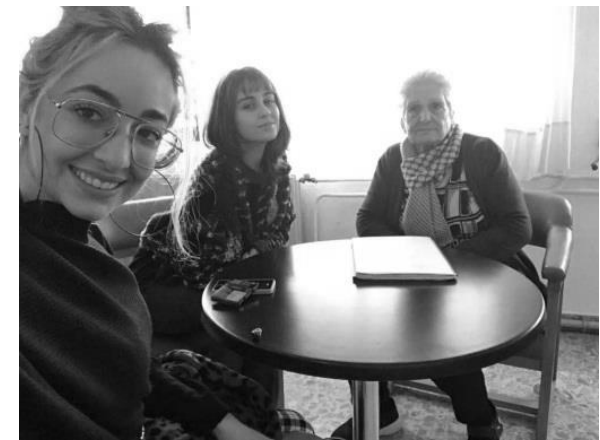

Figura 3. Elaboración de los Relatos de vida.

$3^{\text {a }}$ Progreso en la escena del planteamiento del texto, que se iba elaborando conjuntamente entre el estudiante y la persona mayor. $4^{\mathrm{a}}$ Desarrollo y creación del nudo del relato; $5^{\mathrm{a}}$ Elaboración del desenlace final de la historia. Para lo cual, el alumnado consideró las características del personaje seleccionado, el ambiente en el que se desarrolla la acción, y el cierre.

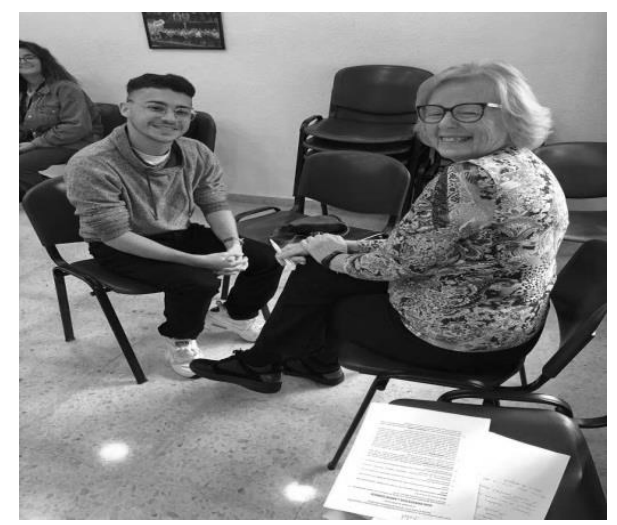

Figura 4. Proceso de realización del planteamiento, nudo y desenlace.

$6^{\mathrm{a}}$ En el propio centro social, se presentó el cuento a la persona mayor, para poder obtener retroalimentación, y de esta manera terminar de ajustar el contenido y una percepción coherente del relato. El soporte utilizado para la edición del cuento fue en papel, utilizando diversos elementos para su encuadernación.

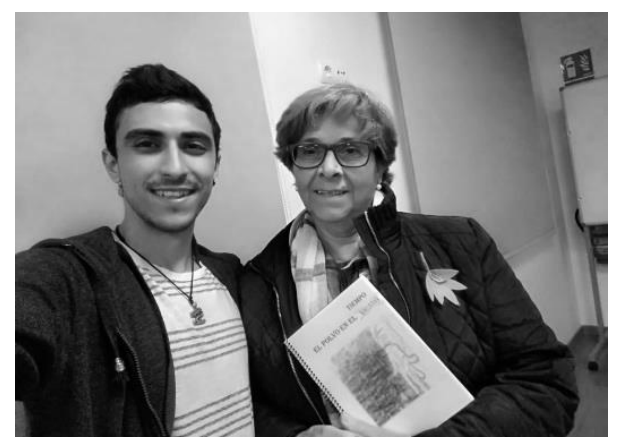

Figura 5. Edición del relato intergeneracional.

Finalmente, se realizó la asamblea evaluativa, tal y como lo describen Martínez de Miguel y Escarbajal de Haro (2009). Los núcleos temáticos giraron en torno a cuestiones curriculares del Grado de Educación Social. Se propusieron las cuestiones bien delimitadas sobre las que reflexionar, y se recogieron los datos en una matriz de análisis tipo DAFO (Sisamón, 2012). De igual forma, se realizó el análisis de los diarios de campos.

\section{Resultados}

El proyecto considerado de manera global implicó: 3 asignaturas, 4 profesores, 2 profesionales del centro de mayores, y 25 usuarios del centro de personas mayores Murcia II, así como 50 alumnos universitarios, divididos en dos grupos.

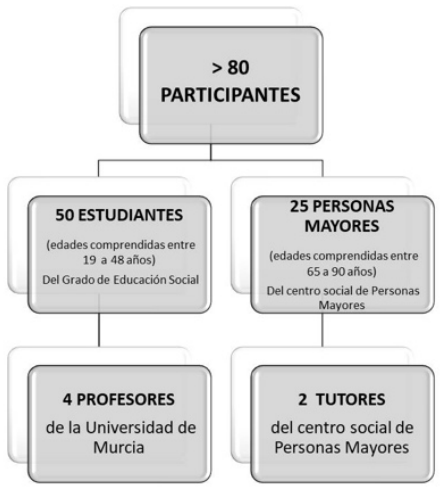

Figura 6. Participantes del proyecto.

Así, respecto a la participación de diferentes asignaturas o programas para este proyecto, cabe señalar que, se estableció una continuidad didáctica en la asignatura de Desarrollo Comunitario y Animación Socicocultural impartida en el primer cuatrimestre del año académico. Los discentes pudieron dar continuidad a sus elaboraciones en la asignatura de Educación de Personas Mayores, impartida en el segundo cuatrimestre, mediante la elaboración de alternativas socioeducativas para personas mayores. De tal forma, que la actividad planteada (relatos de vida), sirvió como base para el análisis de la realidad, y determinación de necesidades o problemas del colectivo de personas mayores. Al tiempo que, el alumnado matriculado en la asignatura de Educación en personas adultas y mayores del Máster de Exclusión e Inclusión social, fue invitado a la discusión y reflexión de los resultados obtenidos.

Por otra parte, y dando respuesta al primero de los objetivos planteados: aplicar una metodología participativa $y$ significativa para el alumno enfocados al desempeño profesional en el centro social de personas mayores, se obtuvieron los siguientes datos tras el análisis DAFO:

\section{Fortalezas}

1. Adquisición de competencias metodológicas.

2. Profundización para elaborar Relatos de vida.

3. Creación de vínculos afectivos intergeneracionales.

4. Conocimientos, experiencias y acercamiento al colectivo.

\section{Oportunidades}

1. Realización de prácticas fuera del aula.

2. Conocer los centros sociales de personas mayores. 
3. Romper estereotipos hacia el colectivo de mayores.

4. Adquirir sabiduría, consejos y puntos de vista diversos.

5. Aumentar habilidades sociales, aceptación de la realidad y conocer modelos de resiliencia.

Amenazas

1. Solapamientos con prácticas en otros centros.

2. Problemas de desplazamientos hasta el centro.

3. Disponibilidad de participantes mayores.

\section{Debilidades}

1. Organización previa y primera en la toma de contacto.

2. Déficits de formación para elaboración de relatos.

3. Mayor número de sesiones.

Tras el análisis de los diarios de campo empleados por el alumnado, los resultados obtenidos fueron analizados vinculándolos a los objetivos propuestos. Así, respecto al segundo objetivo: creación de materiales docentes colaborativos que favorezcan la motivación por el aprendizaje, todo el alumnado consiguió concretar el proyecto diseñado. Fueron elaborados cuentos educativos intergeneracionales en base a los relatos de vida, cuyas acciones se vincularon con competencias y contenidos curriculares referidos a la interacción con la realidad que viven las personas mayores. Respecto del proceso de elaboración de los cuentos intergeneracionales, algunos alumnos mostraron dificultades para afrontar el inicio de la escritura del relatos, si bien su inquietud y motivación hicieron que, finalmente sus escritos adquieran la claridad en la exposición de ideas necesarias. De la misma forma, todos los alumnos mostraron su orgullo por las realizaciones finales conseguidas.

Respecto al tercer objetivo: evaluación de la utilización de estrategias cualitativas intergeneracionales en enseñanza superior por parte del alumnado, se pueden observar dos líneas de actuación fruto de la metodología planteada para el trabajo entre el alumnado y los mayores. Por un lado, todos los alumnos señalaron que se originaron valores de convivencia, y espacios para la participación intergeneracional, con una clara intención profesionalizante. Por otro lado, el alumnado ha subrayado el hecho de que, la experiencia en el trabajo educativo con personas mayores, les ha provisto de conocimientos acerca de la utilización de estrategias socioeducativas de tipo cualitativo. Incluso, aquellos alumnos que inicialmente se mostraban más reacios al contacto con personas mayores, finalmente refirieron su enriquecimiento tanto personal como profesional, para el abordaje de las temáticas gerontagógicas a trabajadas con cada sesión.

\section{CONCLUSIONES}

Para concluir, cabe señalar que con este trabajo se constata el echo de que, este tipo de experiencias posibilitan la ampliación de metodologías colaborativas y participativas para el alumnado. Permiten oportunidades para acercar diferentes áreas curriculares, a la vez que facilitan la fusión de contenidos teóricos con la realidad profesionalizante en un entorno socioeducativo concreto, el de las personas mayores.

Respecto a los aspectos metodológicos alcanzados con la experiencia cabe reseñar, que favorecen la toma de conciencia sobre el aprendizaje por parte del alumnado, así como conecta los conocimientos adquiridos en el aula con la realidad social y el entorno más próximo. De la misma manera, el alumnado se siente identificado con la posibilidad de diseñar, planificar y desarrollar acciones de participación, y de convivencia con diferentes grupos comunitarios. Todo ello, les provee de una autonomía en la acción, facilitadora de empoderamiento, tanto personal como profesional. Posibilita el desarrollo de una acción completa del ciclo de intervención socioeducativa desde una plataforma sociocrítica. Finalmente, les hace sentir protagonistas de la acción educativa, a la vez que parte de la intervención.

En consonancia con lo expuesto anteriormente, el proyecto entronca con las posibilidades educativas intergeneracionales referidas en el apartado de la introducción. Hace protagonista al alumnado en la indagación de contenidos por medio de su conexión intergeneracional en las distintas fases de la experiencia presentada. De esta manera, el alumnado es capaz de aplicar una metodología participativa y significativa. Se consigue realizar diferentes cuentos intergeneracionales, así como se elaboran materiales de trabajo intermedio, que finalmente sirven de soporte para la elaboración de los relatos finales. Además, la experiencia ha posibilitado la motivación e incorporación de los participantes. Todo ello a través de las propuestas realizadas mediante la utilización de estrategias cualitativas, que se relacionan con las competencias básicas de su currículum. Lo cual pudo quedar constatado y reflejado en los diarios de campo.

La exploración de alternativas educativas a partir de procedimientos extraídos y elaborados en contacto directo con los centros de mayores, promovió la capacidad de creación artística del alumnado. Les facilito diferentes formas de abordaje y elaboraciones literarias, a la vez que utilizaron materiales formativos con fines de escritura narrativa. Todo ello les permitió conocer de primera mano las necesidades y problemas, que rodean el ámbito de las personas mayores. De esta manera, se establecieron lazos profesionales y personales de carácter intergeneracional, que permitió desarrollar y estimular sus capacidades para para afrontar contenidos nuevos.

De hecho, la posibilidad de transferencia y aplicabilidad del proyecto a otros entornos, titulaciones o asignaturas también es importante ya que sus elaboraciones, se pueden utilizar como recursos educativos en centros escolares de Educación Primaria y Educación Secundaria, a través del uso de la acción tutorial, así como para los estudiantes de Grado y de Posgrado de la Facultad de Educación, extendiendo la experiencia a nivel comunitario. Respecto a la continuidad del mismo, cabe destacar las sinergias establecidas con otros proyectos desarrollados en el contexto de los centros sociales para personas mayores de la Región de Murcia, como el centro de mayores Murcia II, que presentan unas características idiosincráticas específicas, lo cual facilita alternativas y diversidad en la acción socioeducativa. Todo ello, sin menoscabo de su progresión hacia los próximos años académicos, para los que se mantiene una percepción optimista respecto a su trazabilidad y posibilidades de ejecución.

\section{AgRAdecimientos}

La logística de este trabajo no habría sido posible sin la colaboración de usuarios, y responsables del centro de 
mayores Murcia II. En este sentido, es remarcable la colaboración ofrecida por Basilio Camacho Pérez-Muelas, y Carmela García De Frutos. Asimismo, para el desarrollo y fundamentación teórica del proyecto, cabe destacar el inestimable apoyo del Profesor Andrés Escarbajal De Haro. Finalmente, ha sido providencial la implicación desplegada por los estudiantes del Grado de Educación Social de la Universidad de Murcia a lo largo de todo el año académico. Por todo ello, se considera oportuno refrendar su agradecimiento.

\section{REFERENCIAS}

ACCEM (2018). Escritura creativa. Gramática de la fantasía. Documento policopiado. Recuperado de https://www.accem.es/

Bedmar, M. y Montero, I. (2003). La educación intergeneracional. Un nuevo ámbito educativo. Madrid: Dykinson.

Bolívar, A., Domingo, J. y Fernández, M. (2001). La investigación biográfica narrativa en educación. Enfoque y metodología. Madrid: La muralla.

Delgado, A. y De Justo, E. (2018). Evaluación del diseño, proceso y resultados de una asignatura técnica con aprendizaje basado en problemas. Educación XXI, 21 (2), 179-203. https://doi.org/10.5944/educxx1.19415.

Martín García, A. V. (1995). Fundamentaron teórica y uso de las historias y relatos de vida como técnicas de investigación en pedagogía social. Aula, 7, 41-60.

Martínez de Miguel, S. y Escarbajal de Haro, A. (2009). Alternativas socioeducativas para las personas mayores. Madrid: Dykinson.

Martínez De Miguel, S., Salmerón, J. A. y Escarbajal De Haro (2017). El proyecto intergeneracional universitario. En S. Martínez De Miguel, P. Moreno y A. Escarbajal De Haro (Edits.), Envejecimiento activo, programas intergeneracionales y educación social (pp. 147-174). Madrid: Dykinson.

Martínez De Miguel, S., Salmerón, J. A. y Moreno, P. (2018). El impacto de las acciones intergeneracionales en la enseñanza universitaria. Evaluación de un proyecto universitario a través de sus protagonistas. Personas mayores y estudiantes del grado de educación social. En P. Miralles y C. Romera (Edits.), Metodologías docentes innovadoras en la enseñanza universitaria (pp. 36-49). Murcia: Editum.

Jarré, D. (2016). Tomando decisiones para una sociedad para todas las edades. Bruselas: Unión Europea.

Ramos, J. (2017). Innovación social en la Unión Europea. Redur, 15, 75-84

Rodari, G. (2016). Gramática de la fantasía. Introducción al arte de contar historias. Barcelona: Labutxaca.

Salmerón, J. A., Martínez De Miguel, S. y Belchí, R. (2018). Los proyectos intergeneracionales universitarios con estudiantes de educación social. Estado de la cuestión durante los 10 últimos años. En J. J. Gazquez, et al. (Comps.), Libro de actas del $V$ Congreso Internacional en contextos psicológicos, educativos y de la salud (pp. 224-225). Almería: Universidad de Almería.

Salmerón, J. A., Escarbajal De Haro, A. y Martínez De Miguel, S. (2018). Estudio sobre una experiencia educativa con mujeres mayores en centros sociales. Implicaciones para el aprendizaje a lo largo de la vida. Revista complutense de educación, 29 (2), 317-334. https://doi.org/10.5209/RCED.52381

Sánchez, M., Butts, D. M., Hatton, A., Henkin, N. A., Jarrot, S. E., Kaplan, M.,...Weintraub, A. (2007). Programas intergeneracionales. Hacia una sociedad para todas las edades. Barcelona: La Caixa.

Sisamón, R. M. (2012). El análisis DAFO aplicado a la intervención en casos de personas en situación de exclusión social. Revista de trabajo y acción social, 51, 469-487.

Tamayo Valdés, M. C. y León Pérez, M. Á. (2017). La narración oral. Tradición histórico-cultural y recurso didáctico. Revista Conrado, 13(60), 32-37. Recuperado de http://conrado.ucf.edu.cu/index.php/conrado 